\title{
TRANSITANDO A LA ECONOMÍA CIRCULAR EN EL SECTOR AGROPECUARIO: GRANJAS EXPERIMENTALES EN GUANAJUATO
}

\author{
Gemma Cervantes \\ Universidad De La Salle Bajío, México. Facultad de Agronomía
}

\begin{abstract}
Resumen: El sector agropecuario a nivel mundial, y a nivel de Latinoamérica y de México en específico, es muy importante por el aumento creciente de población, y por tanto de las necesidades de alimentación, pero es uno de los sectores que más funciona con un sistema de producción lineal, en vez de circular. Esto provoca que se genere una gran cantidad de residuos, que por su mala disposición puede provocar contaminación al agua, al suelo y muy especialmente atmosférica. Por tanto, es imprescindible ayudar a que los sistemas agropecuarios tiendan a cerrar el ciclo de materia y así disminuir sus impactos ambientales y obtener un mayor rendimiento en el sistema. Todo ello contribuirá a conocer cómo contribuir a la economía circular desde el sector agropecuario. En este artículo se presenta el estudio hecho con tres granjas experimentales en el estado de Guanajuato (México). Se diagnosticaron cualitativa y cuantitativamente las granjas, se detectaron los aprovechamientos de residuos ya existentes en las granjas y se propusieron nuevos aprovechamientos para cada una de ellas. Con todo ello se propuso una red ecoindustrial agropecuaria entre las tres granjas, formada por 11 entidades, 7 diferentes sectores industriales, 8 sinergias existentes, 18 sinergias propuestas, 22 diferentes residuos valorizados o reusados. Con esta red se plantea una solución para 8 de los 10 residuos más problemáticos de estas granjas y se contribuye a cerrar el ciclo de materia en ellas. También se dan criterios para tender a la economía circular en el sector agropecuario.
\end{abstract}

Palabras claves: economía circular, valorización de residuos, granjas experimentales, simbiosis industrial, sector agropecuario. 


\title{
Moving towards circular economy in agricultural sector: experimental farms in Guanajuato
}

\begin{abstract}
The agricultural sector is very important worldwide, in Latin America and Mexico, due to increasing population and therefore the need for food. But this sector works mainly as a linear production system, instead of a circular one. This causes the generation of large amount of waste, which can cause water, soil and, especially, atmospheric pollution if not disposed correctly. It is therefore essential to help agricultural systems to close the material cycle and thus reduce environmental impacts and obtain a higher performance in the system. This will help to knowing how to contribute to the circular economy in the agricultural sector. This article presents the study carried out with three experimental farms in the state of Guanajuato (Mexico). The farms were diagnosed qualitatively and quantitatively, the valuation of wastes already done in the farms was detected, and new valuations were proposed for each of them. An eco-industrial agricultural network was proposed between the three farms, made up of 11 entities, 7 different industrial sectors, 8 existing synergies, 18 proposed synergies, 22 different recovered or reused waste. With this network, a solution is proposed for 8 of the 10 most problematic farm wastes and contributes to closing the material cycle in them. Criteria are also given to tend to the circular economy in the agricultural sector.
\end{abstract}

Keywords: Circular economy, waste valuation, experimental farms, industrial symbiosis, agricultural sector.

\section{Gemma Cervantes}

Doctora en Química por la Universitat de Barcelona. Su línea de investigación, desde 1999, es ecología industrial y medida de la sustentabilidad. Cuenta con más de 40 publicaciones, entre artículos, libros y capítulos de libros. Es miembro del Sistema Nacional de Investigadores de México, coordinadora de la Red Mexicana de Ecología Industrial, así como profesora e investigadora en la Facultad de Agronomía de la Universidad De La Salle Bajío, México.

Correo: gemma.cervantes@gmail.com

https://orcid.org/0000-0003-1713-3005 


\section{Introducción}

Tanto a nivel mundial como a nivel de Latinoamérica, no se usan los materiales eficientemente, sino que se da una producción y consumo lineal, desechando los residuos y generando nuevas materias primas a partir de la extracción de recursos naturales. Las iniciativas mundiales de ecología industrial (EI) (Ehrenfeld 2004), consumo y producción sustentable (PNUD 2015), decrecimiento (Latouche 2008), enfoque de ciclo de vida (UNEP 2004), economía circular (EC) (Geissdoerfer et al. 2017), entre otras, plantean un cambio hacia un cierre de ciclo de materia donde se tenga en cuenta el ciclo de vida del producto o proceso, donde el residuo sea considerado como una materia prima, donde se disminuya la extracción de recursos naturales y donde se observe la realidad como un sistema en red interrelacionado con lo que le rodea.

Aunque tradicionalmente la agricultura en los pequeños sistemas familiares y rurales se daba como ciclo cerrado donde todos los materiales, residuales o no, eran susceptibles de ser aprovechados, en una economía de autosuficiencia este comportamiento cambió con la llegada de grandes explotaciones y multinacionales en el sector agroalimentario. Actualmente, el sector agroalimentario es uno de los que menos residuos aprovecha, funcionando mayoritariamente como un sistema lineal (FAO 2017).

En Latinoamérica, el sector agropecuario es aún de gran importancia y desde 2015 ha estado creciendo en la mayoría de países, exceptuando en los que ha habido desastres naturales importantes.

Aunque las exportaciones están decreciendo respecto a 2015, desde 2016 empezaron a recuperarse, por lo que podría afirmarse de manera general que el sector agroalimentario está en crecimiento en Latinoamérica (CEPAL et al. 2017). En México, el sector agropecuario también está en crecimiento desde 2016 y sus exportaciones agrícolas están aumentando, destacando el aguacate, con un 46\% del mercado mundial en 2017. Y en el estado de Guanajuato, donde se realizó el estudio que se presenta, el sector agropecuario es uno de los tres más importantes. En 2018 Guanajuato ocupó el octavo puesto a nivel de todo México en producción agrícola (Vizcaino 2019). Por otro lado, en este estado, la mayoría de las explotaciones agropecuarias son pequeñas granjas, muchas de ellas familiares. La FAO y SAGARPA (Secretaría de Agricultura y Desarrollo Rural) han estimado que la agricultura familiar en México, formada por 5.4 millones de Unidades Económicas Rurales (UER), representa el 81.3\% de todas las UER del país (FAO y SAGARPA 2012). 
Por todo lo descrito, se consideró que las propuestas de cierre de ciclo a nivel de pequeñas explotaciones agrícolas pueden llegar a ser un modelo para estos sistemas para tender hacia la economía circular. Se estudiaron tres granjas experimentales que tiene la Universidad De La Salle Bajío en el estado de Guanajuato, llamadas CADELS (Centro Agropecuario de Experimentación De La Salle Bajío) (UDLSB 2020), las cuales tienen diversidad de actividades, como cultivos, diferentes tipos de ganado y animales de granja, compostaje, etcétera. Estas granjas no disponen de una diagnosis cuantitativa, ni un sistema adecuado de gestión de residuos. Tampoco tienen un planteamiento organizado de red entre los centros experimentales. Por otro lado, tienen algunos residuos a los que no están sacando todo su potencial, como material que puede ser reusado, ni su potencial económico, y otros que no saben cómo aprovechar, ni siquiera disponer. Por lo que se propuso una red ecoindustrial en estos sistemas agropecuarios, con intercambio de residuos, como materias primas, que puedan ayudar a tender hacia una economía circular.

Como objetivos específicos se planteó: 1) desarrollar un diagnóstico cuantitativo de las tres granjas experimentales; 2) descubrir las sinergias existentes en las granjas; 3) proponer nuevas valorizaciones de residuos y otros intercambios entre granjas y 4) proponer una red ecoindustrial entre las granjas que pueda contribuir a la economía circular.

\section{Antecedentes y marco teórico}

\subsection{Definiciones de los sectores}

El sector agropecuario es la parte del sector primario, compuesto por el sector agrícola (agricultura) y el sector ganadero o pecuario (ganadería). El sector agroalimentario se refiere a los bienes o servicios relacionados con los productos del campo que se utilizan para la alimentación humana, directamente o después de su elaboración en diversos procesos en la industria alimentaria. Quedan excluidas la acuacultura y pesca (COFECE 2014).

\section{- Importancia del sector agropecuario a nivel mundial y de Latinoamérica}

El sector agropecuario es a nivel mundial un sector clave, por su producción, por la importancia en la alimentación de las personas y por el nivel comercial que genera. Según la FAO, el crecimiento del sector agropecuario en las economías emergentes es y seguirá representando en los próximos años más del doble de la tasa de crecimiento proyectada para los países desarrollados (CEPAL et al. 2019). En el periodo 2008-2018, las exportaciones agroalimentarias de América Latina y el Caribe hacia China aumentaron de $5.6 \%$ a $13 \%$ del total de las exportaciones de la región latinoamericana (Spanish.xinhuanet.com 2018). 


\section{- Importancia del sector agropecuario a nivel de México y Guanajuato}

En México, el sector agropecuario también está en crecimiento desde 2016. Guanajuato es uno de los estados agrícolas más importantes del país. En 2018 ocupó el octavo lugar a nivel nacional en cuanto a producción agrícola, con más de 1,500 millones de dólares en productos agrícolas exportados (Vizcaino 2019). El nivel de exportación registrado durante 2018 confirmó al sector agroindustrial como el motor del desarrollo económico del estado de Guanajuato y lo coloca en segundo lugar en exportaciones, solo por detrás del sector automotriz. En 2016 Guanajuato generó 9.9 millones de toneladas de productos agropecuarios y acuícolas, lo que represento un valor económico de 2,142 millones de dólares, cifras que lo sitúan como unos de los primeros estados a nivel nacional en cuanto a la producción agropecuaria. En bienes pecuarios, la entidad ocupó el cuarto lugar nacional, con una producción de 1.2 millones de toneladas de productos como carne, leche y productos lácteos. Las principales actividades pecuarias son la producción de carne de ave, de porcino y bovino, leche de bovino, y producción de huevo (Sánchez 2017).

Está previsto que este sector experimente crecimiento después de la entrada en vigor (1 de julio de 2020) del Tratado entre México, Estados Unidos y Canadá (T-MEC) (Gobierno de los Estados Unidos de América et al. 2020), con el que se sustituyó el Tratado de Libre Comercio de América del Norte, por lo que aumentará más la importancia del sector agropecuario.

\subsection{Problemática del sector agropecuario}

De acuerdo a la FAO, un 33\% de los alimentos producidos para el consumo humano se pierde o desperdicia en el mundo, lo que representa unos 1,300 millones de toneladas de alimentos al año. Se entiende como Pérdida de Alimentos (PDA) a la merma de alimentos en cualquier parte de la cadena de suministro (producción, post cosecha, almacenamiento o procesamiento de alimentos), es decir, antes de llegar a su fase de producto final (Eguillor 2019), por lo que involucra a las pérdidas en el sector agropecuario. En el caso de Latinoamérica, a pesar que es la región que menos PDA presenta, un $6 \%$ del total mundial, eso supone la pérdida de unos 127 millones de toneladas de alimentos aptos para el consumo humano al año (FAO 2017).

Otra problemática importante es la no adecuada disposición de los residuos pecuarios. Cuando el estiércol de los animales se deja directamente en los campos, o solo se acumula sin compostear, se genera una gran cantidad de metano. El metano tiene potencial para crear efecto invernadero en una tasa 21 veces mayor que el dióxido de carbono (United Nations Climate Change 2020).

Los plásticos residuales del sector agrario son también un problema que ha ido creciendo en los últimos años, pues cada vez más se cultivan especies en climas tradicionalmente no aptos para ellos, gracias a la creación de invernaderos. 


\subsection{La economía circular, la simbiosis industrial y la ecología industrial}

La ecología industrial es el área del conocimiento que busca que los sistemas industriales tengan un comportamiento similar al de los ecosistemas naturales, transformando el modelo lineal de los sistemas productivos a un modelo cíclico, impulsando las interacciones entre la economía, ambiente y sociedad, e incrementando la eficiencia de los procesos industriales (Cervantes 2013). La ecología industrial utiliza la simbiosis industrial (SI) como método. La SI crea redes entre industrias usando los residuos de una industria como materia prima de otra o creando otras sinergias, como pueden ser la compartición de un servicio o infraestructura entre varias industrias. También puede verse la SI como una organización funcional/estructural de un determinado sistema industrial, en que las empresas/agentes involucrados interactúan mutuamente intercambiando los diferentes flujos residuales como materia prima para conseguir un sistema productivo más limpio (Boons et al. 2014). Con la SI se generan asociaciones entre diversas industrias para que se aprovechen los residuos de una como insumos de otra, de modo que ambas se beneficien, con esto se promueve el reciclaje de materiales y energía e indirectamente la creación de redes de información y la innovación tecnológica (Carrillo 2009).

La ecología industrial aplica criterios de desarrollo sustentable como cierre de ciclos de materia con el fin de eliminar los residuos, reducción de los costos ambientales, aumento del capital social local, optimización del uso de materiales residuales concibiendo sinergias, desmaterialización de la economía y la obtención de la mayor parte de energía de fuentes renovables (Carrillo 2013).

Por otro lado, la economía circular es la aplicación de los principios de la ecología industrial a nivel de un sector, región o país, de manera que se tienda a cerrar el ciclo de materia en ese territorio (Kirchherr et al. 2017). De tal manera que hay países que adoptan políticas de economía circular cuando extienden la aplicación de los criterios de ecología industrial a todo el país. China inició la «Política nacional de economía circular» en el año 1999 y en 2009 creó la primera ley de economía circular que existe en el mundo. Actualmente, muchos países a nivel mundial ya han iniciado políticas nacionales de economía circular, entre ellos varios de Latinoamérica.

\subsection{Sistemas ecoindustriales agrarios en México}

Existen ejemplos de sistemas agrarios ecoindustriales en México, que son sistemas que tienden a cerrar el ciclo de materia, en la línea de la economía circular: la granja orgánica Xochimancas (CDMX) tiene caballos, vacas, invernaderos para producción de hortalizas, producción de diferentes compostas, temascal y envasado de hortalizas. En ella existen 13 entidades y 14 sinergias de residuos usados como materia prima. Algunas sinergias son: piedra triturada del temascal como aporte de minerales en la producción de hortalizas, calostro de las vacas para elaboración de un fertilizante, composta para producción de vermicomposta 
(Cervantes 2011). Por otro lado, en el sistema agroindustrial Tochtli (Edo. de Mx.), formado principalmente por la explotación de conejos, aunque también disponen de caseta de aves ponedoras y área de producción de composta, se llevan a cabo varias valorizaciones de residuos, entre ellas la de extracción de larva de mosca del estiércol de conejo. Las larvas vivas que se obtienen son usadas como alimento proteico para sustituir 30\% del alimento convencional de las gallinas (Arce 2010).

Otros aprovechamientos observados respecto a residuos del sector agroalimentario son: sangre como fuente de alimento humano y animal, fertilizante, adhesivo o para obtención de energía; grasa para la obtención de jabones, biodiesel, abonos y pomadas; huesos para la obtención de alimentos, gelatina, abono y artesanías; heces como fuente de abonos o energía; pezuñas para la obtención de queratina; contenido gástrico y ruminal como fuente de energía o para abonos, entre otros (Sánchez 2018).

\subsection{Las granjas experimentales de la UDLSB}

Los Centros Agropecuarios de Experimentación De La Salle (CADELS) son una unidad funcional de investigación, extensión y fomento de la actividad agropecuaria. Las instalaciones, agrícolas y ganaderas, tienen maquinaria especializada y están coordinadas cada una de ellas por personal especializado. Son un espacio importante para el aprendizaje de estudiantes, especialmente de las carreras de Agronomía y Veterinaria, y un espacio imprescindible para la investigación. Estos centros pretenden ser un punto en el que converjan miembros del sector empresarial agropecuario, asociaciones y otras instituciones educativas, quienes podrán acudir a conocer, proponer y difundir tecnologías, sistemas de producción y técnicas de administración relacionados con áreas agrícolas y pecuarias. Existen cuatro CADELS: Santa Rosa (SR), La Estancia (LE), San Miguel (SM) y Silao. Todos están situados en el estado de Guanajuato (UDLSB 2020).

\section{Metodología}

\section{- Diagnóstico de las granjas experimentales}

Se realizaron visitas de campo a tres de las cuatro granjas existentes: Santa Rosa (SR), La Estancia (LE) y San Miguel (SM), y se recopilaron datos cualitativos y cuantitativos. Se usó el software de Google Maps para geolocalizar las granjas.

Se realizaron entrevistas con las personas responsables de ellas y con algunos de los trabajadores. En ellas se puso especial atención en conocer la cantidad, uso, procedencia y destino de los insumos y residuos. Otros datos a recopilar son: tipos de productos y de procesos desarrollados, volúmenes de producción, gestión de alimentos, manejo de residuos generados e infraestructuras. Se realizaron diagramas de flujo cuantitativos de cada centro estudiado, donde se identificaron materias primas, residuos, productos, flujos de agua y energía. En los 
diagramas se utilizó la simbología desarrollada por el Grupo de Investigación en Ecología Industrial (Lule y Cervantes 2010).

\section{- $\quad$ Construcción de los diagramas de sinergias existentes}

Se construyó el diagrama de sinergias de cada una de las granjas, identificando cada etapa de los procesos que se dan en su interior como una entidad. Se consideró como sinergia existente la utilización de un residuo de una entidad como materia prima de otra y la utilización de un servicio o infraestructura común, que ya se esté llevando a cabo. Se reflejó en el diagrama la información recopilada en la etapa anterior respecto al destino o uso de los residuos.

\section{- $\quad$ Propuestas de nuevas sinergias}

En base a los insumos y residuos de cada proceso, a los volúmenes de residuos generados y a la localización de los mismos, se plantearon propuestas viables de intercambio de residuos como materia prima de otros procesos. También, en base a las necesidades de infraestructuras y servicios, se propusieron sinergias de servicios e infraestructuras comunes con otras granjas. Con todos estos datos se construyó el diagrama de sinergias propuestas para las tres granjas. En las sinergias se consideró la posibilidad de utilizar no solo los residuos de las granjas, sino también de otras entidades o zonas locales cercanas.

\section{- $\quad$ Propuestas de valorización de residuos}

En base a las cantidades de residuos de los procesos, se realizó una propuesta de valorización de algunos de los residuos más significativo para cada granja. En el caso de SR, fue el estiércol ovino; para LE, estiércol y aguas residuales a través de un biodigestor anaerobio; para SM aún está en proceso. Para la valorización del estiércol ovino se siguió la metodología de la granja Totchli (Arce 2010). En el caso de LE, los cálculos del volumen del digestor, la producción de biogás, la producción de biofertilizante, así como el área a fertilizar se realizaron utilizando las ecuaciones del manual de biogás (Minenergia et al. 2011) y otras referencias bibliográficas (Varnero 1991). La selección de la tecnología del biodigestor se realizó por medio de visitas a dos empresas locales especialistas en la venta de este tipo de unidades (biodigestores). Las empresas participantes fueron Energymet S.A. de C.V. y Kremeg S.A. Estas empresas tienen su casa matriz en León y Silao.

\section{- $\quad$ Propuesta de red ecoindustrial entre granjas}

En base a las sinergias existentes y propuestas en cada granja, se apuntan intercambios de materiales, servicios e infraestructura entre las granjas, que puedan ser el inicio de una red ecoindustrial entre todas ellas. 


\section{Resultados y discusión}

\subsection{El análisis de las granjas}

\subsubsection{Santa Rosa (SR)}

\section{- Descripción del sistema}

El Centro Agropecuario de Experimentación De La Salle Santa Rosa se encuentra al sur del Municipio de León (Guanajuato), a 1,811 m de altitud y con una extensión aproximada de 16 hectáreas.

Las entidades que forman parte de SR son las siguientes: 1) caseta de vigilancia: se encuentra a la entrada del CADELS; 2) bodega-cantina: está prácticamente en desuso; 3 ) invernadero; 4) área de compostaje; 5) estanque; 6) parcela productiva, dividida en una área de 2 ha, dedicada al riego, y otra de 7 ha, que es temporal; 7) estación meteorológica; 8) almacén de estiércol al aire libre; 9) silos (silo 1 de $240 \mathrm{~m}^{2}$, silo 2 de $248 \mathrm{~m}^{2}$ y silo 3, de $230 \mathrm{~m}^{2}$ ) donde se elabora silo a partir de maíz entero triturado; 10) fosa de borregos: donde se entierran las borregas adultas y crías de borregas muertas; 11) encierro de ovinos, con borregas, sementales y crías; 12) estación eléctrica del pozo que trabaja a 112 $\mathrm{kW} / \mathrm{h}$; 13) aljibe; 14) establo de caballos, con 3 caballos; 15) casetas de aves ponedoras (800 gallinas); 16) bodega de avena. También existen otras entidades como la bodega de herramientas, la bodega de agroquímicos, baños y salones, que no se han considerado para el diagrama de flujo.

Los principales flujos de entrada y salida de cada entidad son los siguientes:

1) Caseta de vigilancia, 2) bodega-cantina, 7) estación meteorológica y 12) estación eléctrica del pozo: no tienen flujos de materia destacables.

3) Invernadero: los flujos de entrada son semillas y plántulas, fertilizantes y agua. Flujos de salida: jitomate, acelgas, rábanos y rastrojos.

4) Área de compostaje. En 2016 se obtuvieron unos $380 \mathrm{~kg}$ de composta a partir de partes iguales de restos de verduras, estiércol de borrego, estiércol de vaca, rastrojo de maíz y gallinaza.

5) Estanque: se recoge el agua de lluvia, pero prácticamente toda se va en evaporación e infiltración.

6) Parcela productiva: en 2016 se produjeron 20 ton de chile por ha, ocupando únicamente las plántulas de chile, estiércol y agua.

8) Almacén de estiércol: se almacena el estiércol producido en las diferentes entidades del CADELS y que posteriormente sale para fertilización de parcelas productivas.

9) Silos: se ocupa como materias primas maíz, plástico de alta densidad y llantas. Como producto sale el silo preparado y los residuos son las llantas y plástico estropeados que no puedan reusarse. 
10) Fosa de borregos: el insumo es cal para favorecer la descomposición e inertización de los cadáveres, el producto de la descomposición se queda en la misma fosa.

11) Encierro de ovinos: las entradas son minerales, rastrojo de maíz, silo de maíz, alimento concentrado (sorgo + semilla de algodón), agua, vacuna, desparasitante, agujas, jeringas, aretes de borregos. A las crías también se les proporciona: leche en polvo, concentrado iniciador, alfalfa, yodo, vitamina E, selenito de sodio y hierro dextrano. Los residuos son estiércol, jeringas usadas, costales del alimento y borregos muertos. Los productos son todas las crías machos y algunas crías hembras para la venta.

13) Aljibe: como insumo está el agua del pozo, que se bombea ocho veces al mes y como salida, el agua que llena los tinacos.

14) Establo de caballos: los insumos son alimento concentrado para caballo y agua para beber y para el lavado. El flujo de salida es el estiércol.

15) Casetas de aves ponedoras: los flujos de entrada son 800 pollitos, el rastrojo de maíz y cáscara de arroz para la cama, el alimento concentrado y agua. Las salidas son pollitos y gallinas muertas y la gallinaza.

16) Bodega de avena: El insumo es la avena, proveniente de otro CADELS. Los flujos de salida son avena para la Feria de León y para otro CADELS.

\section{- $\quad$ Sinergias existentes}

En SR se documentaron varias sinergias que ya se están desarrollando (figura 1). Algunas de ellas provienen del uso de algún material ya disponible en el propio centro y otras se llevan a cabo con residuos procedentes de otros lugares externos: 1) las divisiones de los corrales se hacen con mallas residuales de los campus de la Universidad De La Salle; 2) algunos comederos y bebederos de acero inoxidable para animales son los antiguos mingitorios de edificios de la Universidad De La Salle; 3) se utilizan lonas usadas de la Universidad De La Salle para tapar los silos; 4) se utilizan llantas usadas para aguantar el plástico o lona que tapa los silos; 5) con los costales de alimento residuales se fabrican unas cortinas para tapar el establo de borregas.

\section{- $\quad$ Sinergias propuestas}

En el lugar de estudio existen varios residuos que no son aprovechados y que por tanto pueden dar lugar a potenciales sinergias a proponer. Los residuos son: frascos de insecticidas, medicamentos caducos, llantas estropeadas del silo, estiércol (en época que no se planta), leña que cae de los árboles, pieles de borregos muertos, gallinaza (estiércol + plumas + cama). 
Se propusieron nuevas sinergias (figura 1) que cierren el ciclo de materia o potencien el ahorro de recursos materiales y energéticos y que den respuesta a los residuos que no tienen aprovechamiento:

1) Preparación de diversos tipos de compostas, ya que actualmente no se compostea ningún estiércol. Se propone la preparación de una composta a partir de estiércol y residuos de leña, en pilas volteables. Esta composta se podría aplicar a la parcela productiva cuando se vuelva a sembrar. También se podría preparar lombricomposta, a partir de residuos de fruta y vegetales y de la composta elaborada previamente. De ella se extraería la parte sólida, que se podría usar en los trozos de jardines que existen en el Centro y la parte líquida o lixiviado, que se destinaría a la venta.

2) Construcción de un biodigestor, para aprovechar aquellos residuos orgánicos más difícilmente aprovechables como la gallinaza. También se podría usar parte del estiércol si no interesa hacer tanta composta.

3) Vender las pieles de borregos muertos para ser curtidas. León tiene varias curtidurías donde trabajan con este tipo de pieles. Aunque la ganancia por la venta no es muy elevada, es mucho mejor esta opción que la de disponerlo como basura.

4) Llevar las llantas estropeadas que ya fueron reusadas en el silo a empresas que las trituran. El material granulado que sale se puede usar como un componente muy importante de los asfaltos sonoreductores.

5) Extraer las larvas de moscas del estiércol de borregos para ser utilizadas posteriormente como alimento de gallinas. Este es un proceso sencillo, en el que se deja el estiércol unos días al aire libre para que sea inoculado y después de siete días, cuando la larva está en su plenitud, se separa por fotofobia y se obtienen larvas vivas que se pueden dar como alimento proteico de calidad a las gallinas (Arce 2010). Para esta valorización es mejor utilizar estiércol de ovinos o conejos. 


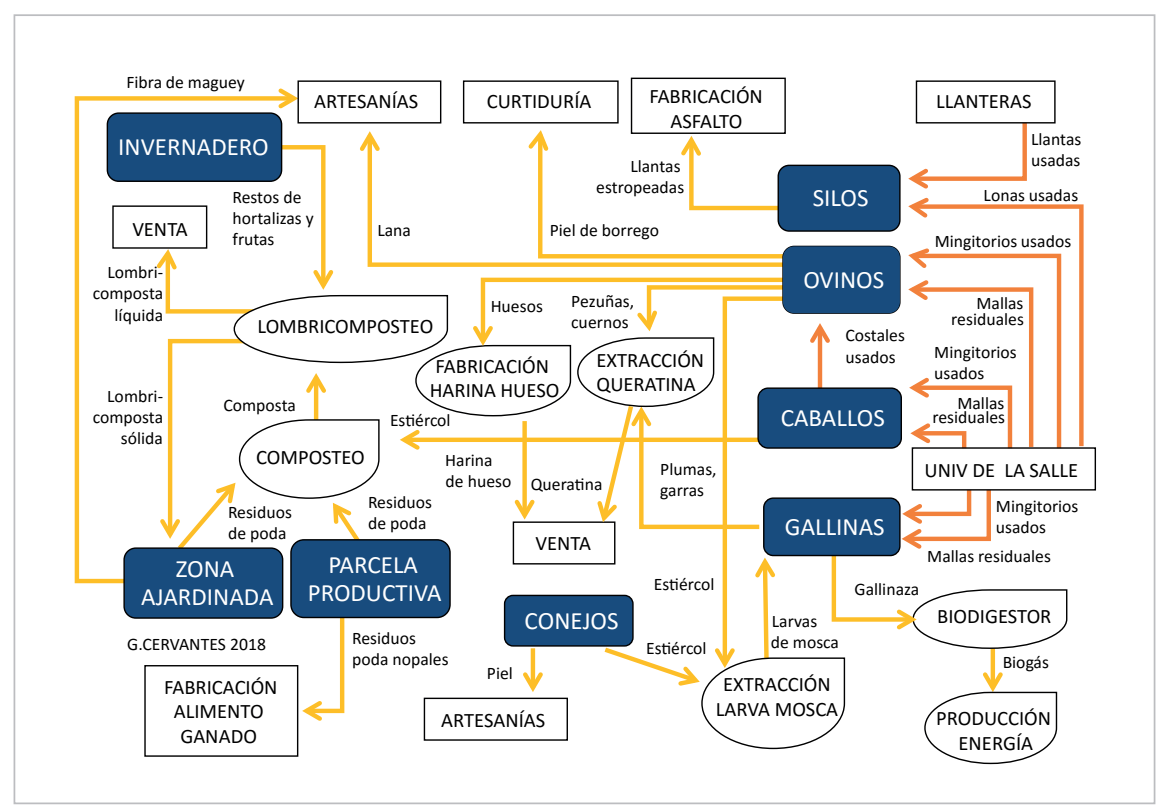

Figura 1: Sinergias existentes y propuestas en la granja experimental SR. Elaboración propia.

\subsubsection{La Estancia}

\section{- $\quad$ Descripción del sistema}

El Centro Agropecuario de Experimentación La Estancia se encuentra ubicado en la Comunidad de La Estancia de los Sapos, en León, Guanajuato, al sur del núcleo urbano y tiene unas 12 ha. Posee 3 hectáreas de infraestructura donde se albergan 2 naves con corrales para el ganado vacuno lechero Holstein. También se dispone de un laboratorio para transferencia de embriones, para lograr mejoramiento genético.

La granja LE tiene 128 animales, de los cuales 45 son vacas lecheras, 15 vacas secas, 20 becerras chicas, 36 becerras grandes y 12 lactantes. Su objetivo es la producción de leche. El alimento del ganado se prepara a partir de forrajes de diferentes especies (avena, alfalfa, maíz, etcétera), los cuales se encuentran almacenados en un área cubierta que está en el interior de la unidad de producción.

El agua que se utiliza en la unidad de producción, se extrae del pozo, se clora y se almacena en un contenedor. Desde este último punto, se distribuye para los diferentes usos dentro de la unidad de producción. El agua se utiliza en el lavado de las ubres de los animales que son ordeñados, lavado de los instrumentos de la ordeña, tanque de almacenamiento de leche, bebederos y sanitarios de los trabajadores. 
La ordeña de los animales que se encuentran en etapa de producción se realiza de manera automática. El producto de la ordeña, leche cruda, se almacena en un tanque refrigerado hasta el momento de su venta. Cuando se vacía este tanque, se lava y se desinfecta para que quede listo para recibir el nuevo lote de producto. En este proceso se utiliza de nuevo agua, la cual termina como agua residual. El caudal de agua residual generada en la unidad de producción es de 2,000 L/día (Barrios 2019) ${ }^{1}$ y se vierte en una fosa séptica antes de ser llevada al sitio de disposición final. El estiércol y orina generados se retiran diariamente de los corrales y se depositan en el patio de la granja, en espera de la llegada de compradores.

Las entidades que se identificaron en LE fueron las siguientes: 1) silo de maíz; 2) almacén de avena y alfalfa; 3) lavadero de casetas individuales; 4) lactancia; 5) almacén de paja; 6) corral de producción; 7) ordeña y tanque de leche; 8) corral de secas; 9) corral de becerras grandes; 10) corral de becerras chicas; 11) ordeña demostrativa; 12) laboratorio de medicamentos; 13) administración; 14) bodega; 15) salón de clases; 16) baños; 17) depósito de agua; 18) estanque; 19) pozo-bomba de agua; 20) caseta de trabajadores; 21) almacén de estiércol al aire libre; 22) fosa séptica.

\section{- $\quad$ Sinergias existentes}

Las sinergias existentes son las siguientes (figura 2): 1) alfalfa, avena y silo preparado del SM para el silo y la alimentación de vacas en LE; 2) lonas usadas de la UDLSB para tapar el silo; 3) llantas usadas de una vulcanizadora para aguantar la lona que tapa los silos; 4) llantas estropeadas del silo son llevadas a una impermeabilizadora que usa llantas trituradas; 5) estiércol que va a una empresa que lo deseca y lo usa en campos de aguacates en Michoacán; 6) punzocortantes usados se llevan a SR donde son dispuestos correctamente como residuos peligrosos.

\section{- $\quad$ Propuesta de nuevas sinergias}

Se propusieron las siguientes sinergias (figura 2):

1) crear un comedor comunitario, con el objetivo de vincularse y favorecer a las comunidades cercanas;

2) hacer composta a partir de los residuos de poda, de las camas de los lactantes (estiércol y paja), de una parte del estiércol del almacén de estiércol y de los retos de comida del comedor comunitario;

3) instalar un biodigestor con una parte del estiércol del almacén de estiércol y con el agua residual de la fosa séptica. Se producirá biogás que puede ser usado en las cocinas del comedor comunitario y también para producción de energía eléctrica;

1 Barrios, R. Veterinario responsable del CADELS La Estancia. Comunicación personal, 3 mayo 2019. 
4) contactar con las comunidades cercanas para que cedan lonas usadas para tapar el silo.

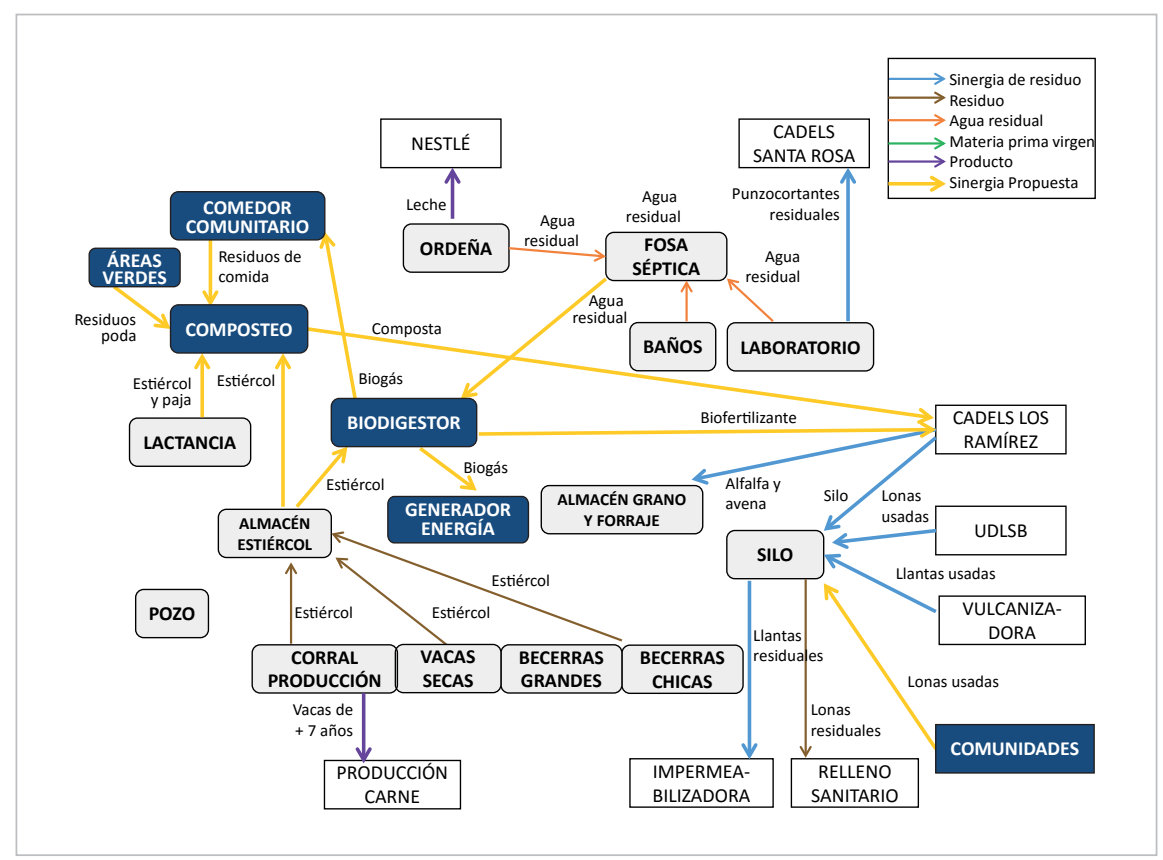

Figura 2: Sinergias existentes y propuestas en la granja experimental LE.

\section{- Descripción de la sinergia: biodigestor}

La unidad de producción de leche LE genera dos tipos de residuos orgánicos, estiércol y aguas residuales, los cuales pueden ser valorizados a través de la construcción y operación de un biorreactor anaerobio (biodigestor) que produzca biogás y biofertilizante. Las ventajas de tener un biodigestor en la unidad de producción es que permite tratar las aguas residuales y estabilizar el estiércol a través de la producción anaerobia de biogás, el cual puede también ser utilizado para la generación de energía (eléctrica y calorífica). Por otro lado, también como subproducto se generan biofertilizantes ricos en materia orgánica y nutrientes (N, P y K), los cuales pueden ser aplicados en los campos agrícolas, disminuyendo de esta manera la cantidad de fertilizantes químicos que se utilizan actualmente y por lo tanto también los impactos ambientales. La cantidad de estiércol generada por día en la unidad de producción es más grande que el caudal de agua residual, por lo cual solo 2,000 kg por día serán utilizados en el biodigestor. La masa de estiércol sobrante podría ser enviada a un proceso de compostaje. 


\subsubsection{San Miguel}

\section{- $\quad$ Descripción del sistema}

El Centro Agropecuario Experimental de la Universidad De La Salle Bajío «San Miguel» se encuentra situado junto a la comunidad de Los Ramírez, en León (Gto.) y tiene 82 ha, de las cuales 71 son cultivables. Del área total, 95\% se dedica a fines comerciales y 5\% para educación e investigación.

Las entidades que forman el sistema son; 1) parcela de maíz de silo, de 27 ha; 2) parcela de maíz de grano, de 40 ha; 3) parcela de alfalfa, de 9 ha; 4) parcela de avena, de 8 ha; 5) parcela de investigación de alumnos, de 4 ha; 6) parcela de investigación del Centro Internacional de Mejoramiento de Maíz y Trigo, CYMMYT, de 2 ha; 7) cuatro pozos y estaciones de bombeo, con una capacidad total de bombeo de $61 \mathrm{~L} / \mathrm{s}$; 8) cuatro estanques, con una capacidad total de 24,000 $\mathrm{m}^{3} \mathrm{y}$ estaciones de rebombeo; 9) huerta de frutales (150 árboles) y jardines; 10) dos invernaderos; 11) área de compostaje; 12) zona de maquinaria, con 29 máquinas; 13) oficinas y 14) zanjas de lluvia y caminos.

En la granja se producen anualmente 2,430 ton de maíz de silo, 600 ton de maíz de grano, 225 ton de alfalfa y 56 Ton de avena. Se dispone de 500 ton de estiércol, traído desde la granja de LE, para la elaboración de composta.

\section{- $\quad$ Sinergias existentes}

Las sinergias existentes son las siguientes (figura 3):

1) alfalfa, avena y maíz de silo para la alimentación de vacas en LE;

2) maíz de silo para elaboración de silo y avena para alimentación de animales en SR;

3) estiércol de LE para la elaboración de composta en SM;

4) frutas residuales, hojarasca y tierra de la huerta de árboles frutales para la elaboración de composta y

5) lonas usadas de la UDLSB para tapar la composta del alumnado. 


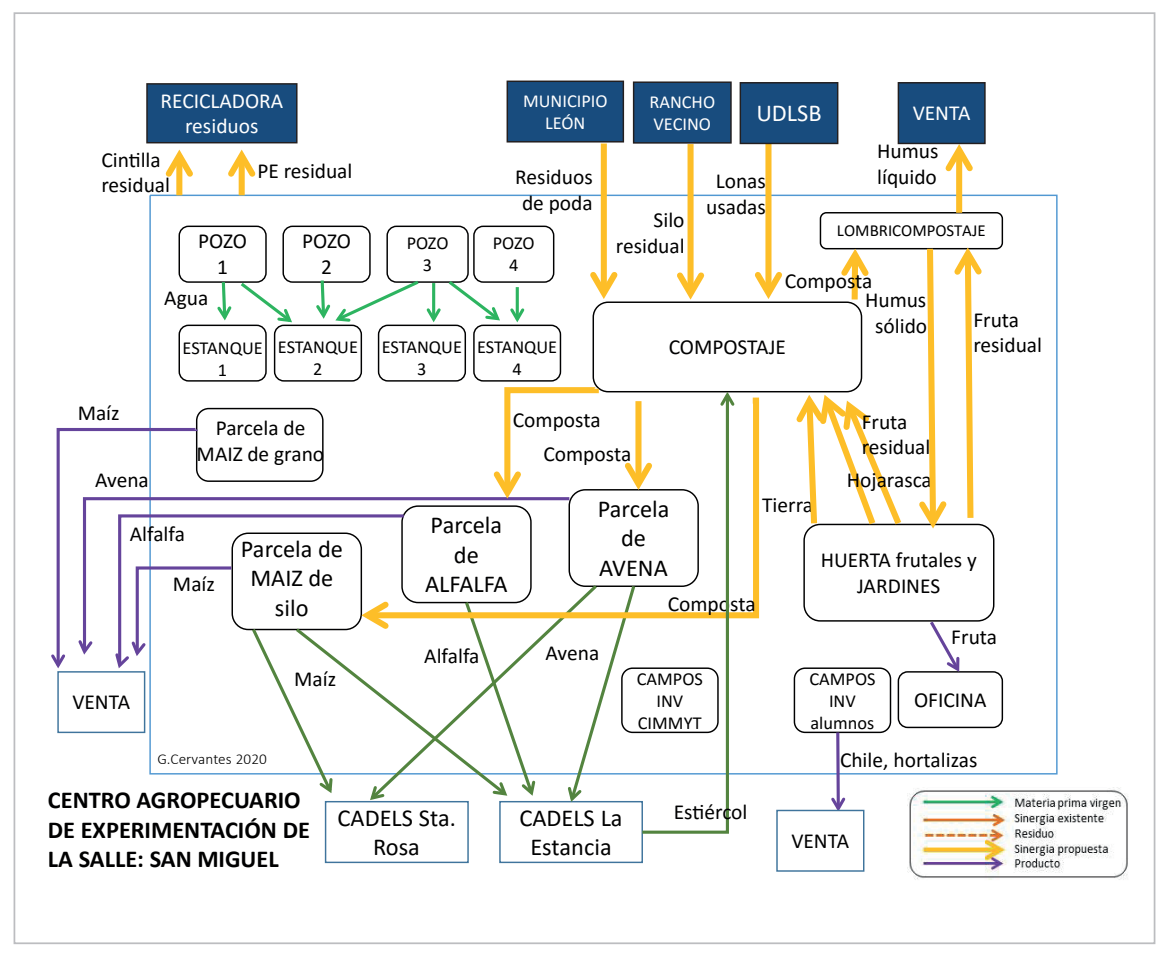

Figura 3: Sinergias existentes en la granja experimental SM.

\subsection{Red de intercambios entre las tres granjas}

Se propuso una red de intercambio entre las 3 granjas, que pueda contribuir a la economía circular. En la red (figura 4) podemos encontrar 4 entidades principales o nodos: la Universidad De La Salle Bajío (UDLSB), la granja SR, la granja LE y la granja SM. También hay otras entidades internas o externas, que ayudan en la valorización de residuos. Estas están marcadas en la figura como pequeños rectángulos. En total se proponen 26 intercambios materiales, la mayoría de ellos constituidos por residuos. No se citan los aprovechamientos que se dan únicamente en el interior de una de las granjas, sin intervención de alguna entidad externa. A continuación, se describen los intercambios, agrupados por entidades:

1) Comedores comunitarios: a ellos se hacen llegar vegetales cultivados en SM, huevos, pollo y carne de cordero procedente de SR y leche de LE. Los residuos orgánicos del comedor van al biodigestor y una parte del biogás generado se usa como gas en las cocinas del comedor. 
2) Biodigestor: el estiércol de las vacas y el agua residual de LE, la gallinaza de SR y los residuos orgánicos del comedor comunitario van al biodigestor. El biogás producido se usa en la cocina de los comedores comunitarios y para calentar agua en LE. También para producir electricidad, que se usa en LE y en SR. El biofertilizante que queda como residuo semilíquido se usa en SM como abono.

3) UDLSB: las lonas usadas de la universidad se usan en SR para tapar el silo y en SM para tapar la composta; con la malla metálica usada se hacen en SR separaciones entre corrales; los mingitorios de acero inoxidable usados se utilizan en SR como comedero y bebedero de animales.

4) SR: las pieles de borregos muertos se venden a curtidurías para ser curtidas; la lana de borregas, las pieles de conejos y las fibras de maguey del área ajardinada se venden para hacer artesanías; las pezuñas y cuernos de ovinos y garras y plumas de gallinas se usan para la extracción de queratina, que posteriormente puede ser vendida; los huesos de ovinos muertos para la fabricación de harina de hueso, que puede ser vendida para fabricar alimento de animales; se extraen las larvas vivas de moscas del estiércol de conejos y borregos para ser utilizadas como alimento de gallinas; las llantas estropeadas que ya fueron reusadas en el silo (por unos diez años) se envían a empresas que las trituran y utilizan el material granulado para asfaltos sonoreductores; los restos de poda de nopales de la zona ajardinada se utilizan para la fabricación de alimento para animales; los residuos de poda de nopales se usan para fabricar alimento de animales para LE; con otros restos de poda se fabrica composta en SM; el silo fabricado en SR se lleva a LE para alimentar a las vacas.

5) LE: actualmente el estiércol se lleva a SM para la fabricación de composta, pero en la red ecoindustrial se propone que se lleve al biodigestor.

6) SM: el maíz de silo y la avena se usan como alimento de animales en SR; el maíz de silo, la avena y la alfalfa se llevan a LE para alimentar a las vacas. 


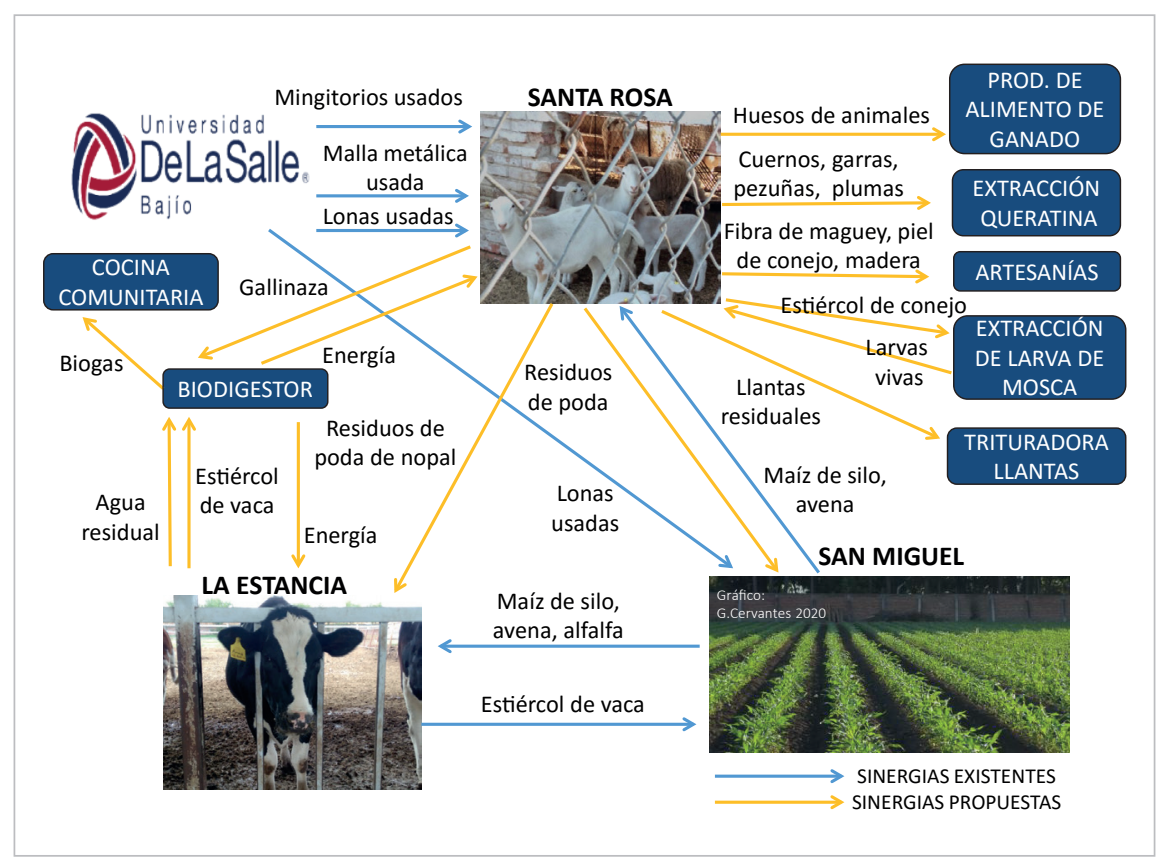

Figura 4: Red Ecoindustrial propuesta para las granjas experimentales SR, LE y SM.

En resumen, esta red está formada por 11 entidades (7 de las cuales son para valorización de residuos), 7 diferentes sectores industriales que intervienen, 8 sinergias existentes, 18 sinergias propuestas, 22 diferentes residuos valorizados o reusados. Con esta red se plantea una solución para 8 de los 10 residuos más problemáticos: estiércol de vaca, estiércol de oveja, gallinaza, agua residual, llantas usadas, pieles de ovejas muertas, huesos de ovejas muertas y residuos de poda. Los botes de insecticida y la medicación caduca no pueden ser reutilizados ni valorizados pues están considerados en la legislación mexicana como residuos peligrosos y deben disponerse en un sitio autorizado. Con esta red se crean 7 nuevos productos que pueden ser vendidos: harina de hueso, material granulado de llantas, queratina, biogás, electricidad, larva viva y artesanías.

\subsection{Propuestas de criterios para tender a la economía circular en el sector agropecuario}

La propuesta de la red ecoindustrial entre granjas experimentales da muchas ideas para cerrar el ciclo de materia en el sector agropecuario, hecho que contribuirá a que se pueda desarrollar una economía circular en la zona.

Los residuos deben ser valorizados en la manera en que se obtengan más productos de él y productos de un mayor valor añadido. Por ejemplo, el estiércol de vaca debe ser llevado a un biodigestor en vez de ser composteado, pues así se obtiene biogás y biofertilizante, o incluso puede obtenerse energía, biogás y 
biofertilizante. Al obtener más productos es más fácil crear interacción con otras entidades y finalmente cerrar el ciclo de materia. También la gallinaza, que es la mezcla de paja y estiércol de gallina, es mejor introducirlo en un biodigestor que compostearlo, pues por su gran acidez y fluidez no es muy adecuado para hacer composta en pilas volteables.

También es recomendable obtener productos innovadores en vez de los tradicionales: por ejemplo, el estiércol de oveja es mejor someterlo al proceso de extracción de larva de mosca, que solo compostearlo, pues así se obtiene proteína viva para alimentar a las gallinas y este producto es una forma innovadora de ahorrar dinero y tener un alimento muy completo para las gallinas.

Las propuestas deben no solo aprovechar un residuo, sino hacerlo de forma que se beneficie al medio ambiente. Por ejemplo, las llantas que ya no se usan, si se dejan en los campos se llenan de agua y son una fuente de mosquitos, al mismo tiempo que no se degradan fácilmente y se quedan por años en el lugar; en cambio, si se trituran puede fabricarse asfalto sonoreductor con ellas y se retiran del campo residuos que estaba perjudicando al medio ambiente.

Es necesario poner imaginación y creatividad para ver recursos y posibilidades donde a veces solo se ven residuos o materiales no aprovechables. Por ejemplo, los cuernos, plumas, garras y pezuñas de animales muertos pueden ser una importante fuente de queratina, la cual se extrae de ellos con un proceso químico sencillo y barato, obteniéndose un producto químico-farmacéutico.

Finalmente, como en la economía circular se entremezclan los aspectos ambientales, económicos y sociales, conviene elegir aquellas valorizaciones o aprovechamientos que más beneficios sociales van a producir. Por ejemplo, la creación de un comedor comunitario cercano a las granjas LE y SR, aparte de permitir que se creen diferentes sinergias, beneficia a los miembros de las comunidades cercanas, que son comunidades con un elevado nivel de pobreza.

\section{Conclusiones}

Con esta propuesta se diagnostican las granjas experimentales de la UDLSB, se da solución a la falta de aprovechamiento de los residuos generados en ellas y se muestran posibilidades de cerrar el ciclo en sistemas agroecoindustriales pequeños. Se proponen valorizaciones para más de veinte residuos y se muestra cómo establecer una red entre sistemas agropecuarios.

Las redes ecoindustriales entre sistemas agropecuarios, que pretenden conseguir el cierre del ciclo de materia, son un buen ejemplo para contribuir a que se pueda desarrollar una economía circular en el sector agropecuario, pues contribuyen, a pequeña escala, al aprovechamiento de los residuos de unas entidades como materia prima de otras entidades.

En el artículo se detallan propuestas para que los sistemas agropecuarios puedan ir tendiendo a la EC, tales como valorizar a los residuos en la forma en que se obtengan más productos de ellos y productos de un mayor valor añadido, obtener 
productos innovadores, elegir las mejores opciones a nivel medioambiental, fomentar el beneficio social etcétera.

Las granjas experimentales son también una excelente oportunidad para investigadores y estudiantes para aplicar fácilmente los métodos y principios de la ecología industrial y de la economía circular a sistemas reales.

Para el caso de México y del estado de Guanajuato, este ejemplo de granjas experimentales puede ser modelo para otros sistemas agropecuarios del estado, pues la inmensa mayoría de sistemas agropecuarios del estado son pequeños.

\section{Referencias}

Arce, J. (2010). Aplicación de criterios de ecología industrial en un sistema agrario (Proyecto Terminal de Ingeniería Ambiental). Instituto Politécnico Nacional: Ciudad de México.

Boons, F., Spekkink, W. y Jiao, W. (2014) A Process Perspective on Industrial Symbiosis. Theory, Methodology, and Application. Journal of Industrial Ecology, 18(3): 341-355. https://doi.org/10.1111/jiec.12116

Carrillo, G. (2009). Una revisión de los principios de la ecología industrial. Argumentos, 22(59): 247-265. http://www.redalyc.org/articulo. oa?id=59511412009

Carrillo, G. (2013). La Ecología Industrial en México. México DF: Ed. Universidad Autónoma Metropolitana.

Cervantes, G. (2011). Ecología Industrial: innovación y desarrollo sostenible en sistemas industriales. Revista Internacional Sostenibilidad, Tecnología y Humanismo, (6): 58-78.

Cervantes, G. (2013). El Concepto de la Ecología Industrial. En G. Carrillo (Ed.), La Ecología Industrial en México (pp.43-64). México DF: Ed. Universidad Autónoma Metropolitana.

COFECE (Comisión Federal de Competencia Económica) (2014). Reporte sobre las condiciones de competencia en el sector agroalimentario. Resumen ejecutivo. https://www.cofece.mx/cofece/images/Estudios/COFECE_resumen_v04_ alta.pdf

CEPAL, FAO y IICA (2017). Perspectivas de la agricultura y del desarrollo rural en las Américas: una mirada hacia América Latina y el Caribe 2017-2018. https://repositorio.cepal.org/bitstream/handle/11362/42281/1/ PerspAgricultura2017-2018_es.pdf

CEPAL, FAO y IICA. (2019). Perspectivas de la agricultura y del desarrollo rural en las Américas: una mirada hacia América Latina y el Caribe 2019-2020. https:// repositorio.iica.int/bitstream/handle/11324/8214/BVE19040295e. pdf;jsessionid=33F647D1DEDB023540FF16D6966EB816? sequence=1 
Eguillor, P. (2019). Pérdida y desperdicio de alimentos en el sector agrícola: avances y desafíos. Febrero de 2019. Santiago de Chile, Chile: ODEPA. Recuperado de: https://www.odepa.gob.cl/wp-content/uploads/2019/02/articuloperdida_desperdicios.pdf

Ehrenfeld, J. (2004). Can Industrial Ecology be the Science of sustainability? Journal of Industrial Ecology, 8(1-2): 1-3. doi:10.1162/1088198041269364

FAO (2017). Pérdida y Desperdicio de Alimentos en América Latina y El Caribe: Alianzas e institucionalidad para construir mejores políticas. Boletín 4. http://www.fao.org/3/a-i7248s.pdf

FAO y SAGARPA (Secretaría de Agricultura y Desarrollo Rural) (2012). Agricultura familiar con potencial productivo en México. http://www.fao.org/3/abc944s.pdf

Geissdoerfer, M., Savaget, P., Bocken, N. y Hultink E. (2017). The Circular Economy, A new sustainability paradigm? Journal of Cleaner Production, 143: 757768. http://dx.doi.org/10.1016/j.jclepro.2016.12.048

Gobierno de los Estados Unidos de América, Gobierno de los Estados Unidos Mexicanos y Gobierno de Canadá (2020). Protocolo por el que se sustituye el tratado de libre comercio de América del Norte por el tratado entre los Estados Unidos de América, los Estados Unidos Mexicanos y Canadá. https:// www.gob.mx/cms/uploads/attachment/file/465881/T-MEC_Protocolo. pdf

Kirchherr, J., Reike, D. y Hekkert, M. (2017). Conceptualizing the circular economy: An analysis of 114 definitions. Resources, Conservation \& Recycling, 127: 221-232. https://doi.org/10.1016/j.resconrec.2017.09.005

Latouche, S. (2008). La apuesta por el decrecimiento. ¿Cómo salir del imaginario dominante? Barcelona: Icaria editorial.

Lule D. y Cervantes G. (2010). Diagramas de flujo de sistemas industriales, una herramienta para la ecología industrial. El caso del corredor industrial de Altamira. En V Congreso Sistemas de Innovación para la Competitividad SINNCO 2010. Consejo de Ciencia y Tecnología del Estado de Guanajuato (CONCYTEG), León.

Minenergia (Ministerio de Energía de Chile), PNUD y GEF (2011). Manual de biogás. Proyecto CHI/00/G32 «Chile: Remoción de Barreras para la Electrificación Rural con Energías Renovables». Santiago de Chile (Chile): FAO.

PNUD (2015). Objetivos de desarrollo sustentable. https://www.undp.org/content/ undp/es/home/sustainable-development-goals/resources.html

Sánchez, A. (2017). Guanajuato en el top 10 de producción agropecuaria. Milenio, 1 abril 2017. Disponible en: https://www.milenio.com/estados/guanajuatoen-el-top-10-de-produccion-agropecuaria

Sánchez, I. (2018). Simbiosis agroindustrial en Pénjamo, Guanajuato, México. Tesis de Licenciatura en Ingeniería Ambiental. Universidad de Guanajuato, Guanajuato. 
Spanish.xinhuanet.com. (20 de mayo de 2018). Especial: Sector agropecuario de América Latina recoge beneficios de relación con China. Xinhua español. Recuperadode:http://spanish.xinhuanet.com/2018-05/20/c_137191824. htm

UDLSB (Universidad De La Salle Bajío) (2020). Centro Agropecuario de Experimentación. http://bajio.delasalle.edu.mx/apoyos/experimental.php 2020

UNEP (2004). ¿Por qué adoptar un enfoque de ciclo de vida? https:// sustainabledevelopment.un.org/content/documents/1731Why_take_a_ life_cycle_approach_ES.pdf

United Nations Climate Change (2020). Global Warming Potentials (IPCC Second Assessment Report). https://unfccc.int/process/transparency-andreporting/greenhouse-gas-data/greenhouse-gas-data-unfccc/globalwarming-potentials

Varnero, M. T. (1991). Manual de Reciclaje Orgánico y Biogás. Santiago de Chile: Ministerio de Agricultura (FIA) y Universidad de Chile.

Vizcaíno A. (3 de abril de 2019). Guanajuato, octavo en producción agrícola. El sol de Salamanca. Recuperado de: https://www.elsoldesalamanca.com.mx/ local/guanajuato-octavo-en-produccion-agricola-3273292.html 